\title{
Mohammed Dib/Jean Sénac, dossier coordonné par H. SANSON et G. DUGAS
}

\section{Emanuela Cacchioli}

\section{(2) OpenEdition Journals}

\section{Édition électronique}

URL : https://journals.openedition.org/studifrancesi/45534

DOI : 10.4000/studifrancesi.45534

ISSN : 2427-5856

\section{Éditeur}

Rosenberg \& Sellier

\section{Édition imprimée}

Date de publication : 1 août 2021

Pagination : $411-413$

ISSN : 0039-2944

Référence électronique

Emanuela Cacchioli, «Mohammed Dib/Jean Sénac, dossier coordonné par H. sanson et G. Dugas », Studi Francesi [En ligne], 194 (LXV | II) | 2021, mis en ligne le 01 septembre 2021, consulté le 15 octobre 2022. URL : http://journals.openedition.org/studifrancesi/45534 ; DOI : https://doi.org/10.4000/ studifrancesi.45534

Ce document a été généré automatiquement le 15 octobre 2022.

\section{(†) $\odot$

Creative Commons - Attribution - Pas d'Utilisation Commerciale - Pas de Modification 4.0 International - CC BY-NC-ND 4.0

https://creativecommons.org/licenses/by-nc-nd/4.0/ 


\title{
Mohammed Dib/Jean Sénac, dossier coordonné par H. SANSON et G. DUGAS
}

\author{
Emanuela Cacchioli
}

\section{RÉFÉRENCE}

Mohammed Dib/Jean Sénac, dossier coordonné par H. SANSON et G. DUGAS, “Europe, revue littéraire mensuelle" 1094-1095-1096, juin-juillet-août 2020, 350 pp.

1 Fondée en 1923 sous l'égide de Romain Rolland, la revue "Europe" consacre depuis toujours ses dossiers à un ou à plusieurs écrivains qui ont désormais atteint une renommée internationale. Le volume, paru au cours de l'été 2020, est dédié à deux auteurs très représentatifs de la littérature algérienne. Il s'agit de Mohammed Dib et de Jean Sénac. C'est Henri SANSON qui coordonne la première partie du volume et qui présente au lecteur la personnalité polyvalente de Mohammed Dib à l'occasion du centenaire de sa naissance. Connu surtout pour ses œuvres romanesques, l'écrivain algérien est décrit dans ce dossier à travers ses nombreuses facettes: les spécialistes apportent leurs contributions à propos de sa production poétique, théâtrale, de ses contes et de ses nouvelles. Ce corpus varié permet de montrer la richesse créative de Dib, ainsi que son travail sur la forme, sur le genre et sur le langage. Les articles aident également à parcourir cinquante ans de carrière d'un écrivain qui a traversé tous les événements majeurs de l'histoire récente de son pays: des guerres, des révolutions, la décolonisation, la ségrégation sociale et la mondialisation. Dans ses œuvres, Dib a toujours gardé le lien avec son peuple et il a participé à ses souffrances et à son combat. Son engagement a comporté la nécessité d'une recherche sans trêve, d'une quête continuelle qui l'a conduit à (se) poser des questions et à refuser toute certitude acquise. Sanson met en relief les caractéristiques de ce dossier: la contribution de spécialistes ayant accompli des parcours d'études différents; les nombreuses approches critiques utilisées, dont la génétique textuelle; la publication de quelques textes inédits qui pourrait stimuler de nouvelles pistes de recherche. Le dossier s'ouvre avec quatre 
témoignages. Frédéric Jacques TEMPLE parle de sa rencontre avec Dib en 1949 et de la publication du poème Temps mort (dont il offre le texte au lecteur) qui est paru pour la première fois dans la revue "Souffles" et qui a été réédité comme Éléments en version augmentée et restructurée en 1961. Albert MEMMI nous rappelle son activité de collaboration pour le journal "Action" et son choix de lui consacrer une page hebdomadaire en 1955, ce qui a permis à Dib de se faire connaître en France. Memmi publie également une lettre que l'écrivain algérien lui a envoyée à l'époque. Habib TENGOUR raconte comment son activité de poète a été influencée par Dib dès sa jeunesse: son désir de liberté, sa revendication d'égalité, ainsi que la nécessité de s'interroger ont été, pour lui, un exemple à suivre. Abdelkader DJEMAÏ nous offre un récit de sa rencontre avec Dib en 1946 quand l'écrivain avait 26 ans et était encore "seulement" un poète car il n'avait pas encore publié ses romans. Bien que d'une longueur inégale, les contributions qui suivent offrent au lecteur un point de vue critique. Naget KHADDA met en relief la recherche esthétique de Dib, le choix d'écrire en français, d'interpréter la parole collective mais avec un ton intimiste, la nécessité d'explorer la condition humaine à partir d'un syncrétisme culturel à même de fusionner plusieurs éléments hétérogènes. Benjamin GUÉRIN consacre son article à l'enfance, c'est-à-dire au mélange d'innocence et de transgression, mais aussi de jeu et de liberté qui caractérise les œuvres de Dib. La contribution de François DESPLANQUES porte sur la beauté des paysages qui représentent la toile de fond des romans de l'écrivain algérien. «Bien plus qu'un simple décor extérieur», le paysage "colore et nourrit sa vie psychique» (p. 41). En cinquante ans de carrière, l'écriture et les situations changent, mais les constantes sont nombreuses: avec acuité et originalité, Dib montre l'immensité, la nudité et l'âpreté de l'Algérie en faisant appel aux cinq sens. Abdellatif LAÂBI aborde le thème de la responsabilité dans les nouvelles du recueil La Nuit sauvage. Denise BRAHIMI analyse l'impact de la modernité dans la société traditionnelle algérienne, telle qu'il est traité dans le roman Si Diable veut. La sagesse populaire et les coutumes ancestrales sont les aspects les plus évidents d'une société complexe qui se trouve dans une situation d'équilibre fragile. Quand la modernité fait irruption dans cet univers, les conséquences peuvent être déstabilisantes, voire dramatiques. L'article de Karolina RESZTAC revient sur la thématique des croyances populaires dans la pièce de 1965, La Fiancée du printemps, et le roman de 1998, Si Diable veut. Bien que le genre littéraire et les moments de la rédaction soient différents, les deux œuvres abordent la même histoire du rite non accompli. Les deux contributions suivantes sont consacrées à Les terrasses d'Orsol, un roman de 1985. Charles BONN analyse les éléments de rupture avec l'époque précédente (le déplacement géographique et la dimension autobiographique), tout en mettant en évidence la continuité thématique et notamment le traitement réservé au langage. Abdellah ROMLI se concentre sur «l'atmosphère singulière de doute et de questionnement» (p.74) à travers une analyse génétique des avant-textes et des dossiers préparatifs qui démontrent la longue gestation et la complexité du texte. Tristan LEPERLIER nous offre une lecture de la nouvelle Une partie de dés, parue dans le recueil La Nuit sauvage. Le conte se déroule dans le cadre spatio-temporel de la guerre civile algérienne des années quatre-vingt-dix. L'étude de la genèse du texte met en relief le travail que Dib a fait pour transformer l'enjeu politique en enjeu psychologique et métaphorique. Par conséquent, on peut constater le passage d'un engagement explicite et polémique à une responsabilité plus universelle. On revient à la poésie avec l'article de Ismail ABDoun. À partir du recueil de poèmes L'Enfant-jazz, le chercheur se 
focalise sur la relation entre littérature et musique et notamment le jazz. Le rythme, mais aussi la structure sont les points communs de ces deux systèmes sémiotiques différents. À propos de la même collection poétique, Claude RIFFARD offre une étude génétique des avant-textes qui démontre l'élaboration ayant précédé la version définitive. Manel AïT MEKIDÈCHE aborde le thème du clonage qui, dans son optique, devient la «manifestation politique du monolithisme de la pensée» et l'«incarnation des altérités hostiles de l'exclusion et du langage totalitaire» (p. 104). Le clonage rend donc évidente la difficulté de donner du sens au présent. Lakis PROGUIDIS se concentre sur Simorgh, un recueil contenant des textes hétérogènes: des mythes, des contes, des extraits de journal, des aphorismes, des chroniques et des critiques littéraires. L'article propose quelques pistes pour retrouver une unité thématique qui est difficile à détecter à une première lecture. Catherine BRUN aborde la question de l'autre, telle qu'elle a été considérée dans L'Arbre à dires, un ouvrage énigmatique qui bouscule entre essai et fiction, entre culture maghrébine et culture française. Salim JAY consacre sa contribution au parcours littéraire de Dib, à sa soif de vérité et à son attitude loyale envers le lecteur. Ce sont les mêmes aspects que l'on retrouve chez Malek Alloula. La section suivante propose des textes inédits de Mohammed Dib. Il s'agit de: Quelques mots à propos de "Qui se souvient de la mer", une postface alternative à celle qui a été publiée plus tard et qui insiste sur la cohérence de l'œuvre et sur la continuité du parcours littéraire de Dib; Jean Pélégri, ou le juste mot à dire, un texte où l'écrivain déplore le manque de reconnaissance pour cet auteur algérien; Jehan Le Simple, un conte inédit, destiné aux adultes. Le lecteur peut également profiter d'un dossier à propos de la pièce inédite, La Fiancée du Printemps, qui se compose d'une présentation d'Hervé Sanson, de quelques notes et d'un résumé de Dib, ainsi que d'un extrait (le premier acte de la dernière version de 1985). Cette première partie se conclut avec une série de contributions courtes qui sont offertes au lecteur par des écrivains, des poètes, des traducteurs ou des photographes qui considèrent Mohammed Dib comme un point de référence incontournable pour leurs créations artistiques. Ce sont Angelica AMMAR, Samira NEGROUCHE, James SACRÉ, Philippe BORDAS, Jean-Pierre CHAMBON, Pierre JORIS, Satoshi UDO qui rendent hommage à l'écrivain algérien avec leurs témoignages ou leurs articles critiques.

2 Le deuxième volet de la revue est consacré à Jean Sénac, un écrivain algérien contemporain de Dib, assassiné en 1973. Guy DUGAS introduit la personnalité de l'auteur et le définit comme un médiateur intellectuel, un citoyen du monde qui a lutté pour l'indépendance de son pays, qui a dénoncé les guerres coloniales, le racisme et l'homophobie par ses écrits et par son action. Bien qu'il ait laissé une production comprenant à la fois du théâtre, des romans et de la critique, c'est surtout pour sa poésie qu'il est connu. Cependant, selon Dugas, Sénac n'a pas obtenu la place qu'il mériterait dans la littérature universelle. Passionné d'art, dessinateur, doté d'une curiosité intellectuelle fine, il entre en écriture après sa rencontre avec René Char. Depuis ce moment, il trouve sa voix poétique et son parcours d'écrivain s'accomplit entre la France et l'Algérie. René DE CECCATTY trace un profil biographique et intellectuel et l'associe à l'écrivain italien Pier Paolo Pasolini. Les affinités sont nombreuses: leur mort mystérieuse, leur homosexualité, leur conception du civisme et leur esprit révolutionnaire, ainsi que leur volonté d'intervenir dans la vie politique les rapprochent. Dominique cOMBE reprend le profil biographique et intellectuel de Jean Sénac et s'arrête notamment sur quelques aspects particuliers: l'absence du père, le 
choix du français comme langue d'écriture imposée (il parle espagnol, mais pas l'arabe), son statut de «poète algérien» (p. 216). Albert BENSoussan rend hommage à «une des voix les plus prodigieuses» de l'Algérie (p. 224) et surtout à sa production poétique. L'article se consacre au lien entre Sénac d'un côté, Char, Rimbaud et Verlaine de l'autre. La contribution de Pierre RIVAS porte sur la «métaphysique du sexe» (p. 225), c'est-àdire sur la relation entre le sexe et le projet de vie de Sénac, voire les cycles de sa biographie et son œuvre. C'est notamment dans ses deux derniers recueils Le Mythe du Sperme-Méditerranée et Dérision et Vertige que le poète aborde cette thématique de façon plus explicite. Selon le critique, cette attitude n'étonne pas car la sexualité remplace la désillusion politique et devient un rempart: après une phase de révolte et de culpabilité, le poète se livre à l'abandon et à la confiance. Abdelmadjid KAOUAH revient sur le profil biographique et littéraire de Jean Sénac et sur son ambition de devenir un écrivain célèbre qui est à l'origine de son déplacement à Paris. Son aperçu met en relief l'engagement de l'auteur algérien et sa rencontre avec Jean-Pierre Bénisti. Éric SARNER rend un hommage poétique à Jean Sénac pour rappeler la nécessité de garder sa mémoire. Kai KRIENKE nous propose un panorama de la réception de l'œuvre de l'écrivain algérien aux États-Unis et il se focalise sur les traductions et sur les travaux universitaires. Le lien avec l'Amérique du Nord s'établit tout seul parce qu'on constate plusieurs convergences entre Sénac, la Beat Generation et la Harlem Renaissance au niveau de la vision politique, sociale et de l'engagement en poésie. L'auteur algérien s'est intéressé aux poètes américains et leur a consacré plusieurs émissions sur Radio Alger. Le critique met en évidence les affinités avec Édouard Roditi et Charles Olson. La contribution suivante rassemble trois messages que Mohammed DIB, YOURI et Emmanuel ROBLÈs ont écrits en 1973, quelques semaines après la mort de Sénac, pour l'inauguration d'une exposition en son honneur. Le dossier se conclut avec quelques inédits. Il s'agit de trois poèmes (Les naufragés de la mémoire, En cet octobre 70, Sur Tigzirt ou....?) et de deux textes en prose (Avertissement et dédicace, pour un recueil de poèmes inédits, et Préface à un diwan). La dernière section de la revue propose plusieurs rubriques contenant des textes ou des articles critiques sur des sujets différents («Cahier de création», «Chroniques», «La machine à écrire», «Les quatre vents de la poésie», «Le théâtre», «Le cinéma», «La musique») et des «Notes de lectures» où nous retrouvons quelques comptes rendus à propos de Mohammed Dib et de Jean Sénac.

La revue "Europe" nous offre un volume riche en contributions diverses et hétérogènes en mesure de montrer l'originalité de deux écrivains algériens qui ne cessent de nous parler. 